$(70 \%)$. Results show no significant relationship between EULAR moderate response or EULAR good response and the presence of comorbidity. Lower HAQ, lower baseline DAS28 and seronegativity contribute towards good treatment response.The limitations of this study include gaps in clinical records, absence of patient reported questionnaires, and small size of database.

Disclosure of Interest: None declared

DOI: 10.1136/annrheumdis-2017-eular.2025

\section{AB0304 SERUM LEVELS OF SCLEROSTIN AND DICKKOPF-1, AND THEIR CORRELATION WITH BONE MINERAL DENSITY AND BONE MARKERS IN RHEUMATOID ARTHRITIS}

C.A. Nava-Valdivia ${ }^{1}$, E. Corona-Sanchez ${ }^{2}$, J. Ponce-Guarneros ${ }^{3}$, A. Saldaña-Cruz ${ }^{2}$, J. Murillo-Vazquez ${ }^{2}$, E. Perez-Guerrero ${ }^{2}$,

P. Hernandez-Cuervo ${ }^{2}$, M. Salazar-Paramo ${ }^{3}$, D. Cardona-Müller ${ }^{2}$

E. Cardona-Muñoz ${ }^{2}$, L. Gonzalez-Lopez ${ }^{3}$, J. Gamez-Nava ${ }^{1} .{ }^{1}$ Unidad Biomédica 02, IMSS; ${ }^{2}$ Universidad de Guadalajara; ${ }^{3}$ MSS, Guadalajara, Mexico

Background: Sclerostin (SOST) and dickkopf-1 (DKK1) are involved in the development of primary osteoporosis. Although to date, it is controverted does exist about if serum levels of these molecules may reflex adequately a subgroup of patients with decrement of Bone Mineral Density (BMD) being useful as clinical biomarkers. Some non-conclusive observations made in postmenopausal osteoporosis have shown abnormalities in serum levels. To date, there is a lack of information about if these serum molecules are associated with a decrease of $B M D$ in RA. This information is relevant for to know if the measurement of these serum molecules could be useful in the clinical care to identify a subgroup of patients with low BMD.

Objectives: To evaluate if serum levels of SOST and DKK1 correlate with bone mineral density and other bone markers in RA.

Methods: In this Cross-sectional study, we included 115 women with RA $>40$ years old. All patients were assessed for clinical characteristics disease activity (DAS28) and functioning (HAQ-Di). BMD was measured in lumbar spine, hip and forearm using DXA. According to their results, osteoporosis was identified if the BMD in a patient had a T-score with a decrement of $<-2.5$ standard deviation (SD). According to their results we classified these patients in two groups: a) low BMD (T-score $\leq-1$ SD on lumbar spine or total hip) and b)normal BMD (T-score $>$ 1SD). Serum SOST, DKK1, including serum Receptor Activator of NF-KB Ligand (sRANKL) and osteoprotegerin (OPG) were quantified by ELISA. We compared serum levels between the groups using Student $t$-tests. For comparisons between proportions we performed Chi-square test (or Fisher exact test if required). Correlations between serum levels of SOST, DKK1, with BMD, clinical variables, sRANKL and OPG we performed Pearson correlation tests.

Results: From the 115 women with RA, 58 patients had low BMD (50.4\%), from them 44 patients $(38.3 \%)$ had osteoporosis. Patients in the group with low BMD had higher age $(63 \pm 8$ vs $53 \pm 9, p<0.0001)$, DAS28 $(4.1 \pm 1.5$ vs $3.3 \pm 1.4, p=0.011)$, and $\mathrm{HAQ}-\mathrm{Di}$ score $(0.69 \pm 0.53$ vs $0.36 \pm 0.47, \mathrm{p}=0.001)$ compared with normal BMD; whereas lower serum levels of sclerostin was observed in the low BMD group $(128.5 \pm 75.0$ vs $178.2 \pm 103.3, p=0.004)$. SOST levels were correlated with BMD in lumbar spine $(r=0.393, p<0.0001)$, hip $(r=0.388, p<0.0001)$ and forearm $(r=0.319, p=0.001)$. sRANKL serum levels had a negative correlation with BMD in hip $(r=-0.17, p=0.03)$. No correlations were observed between serum DKK1 and OPG with BMD in hip or lumbar spine.

Conclusions: These results indicate that SOST, and RANKL are biomarkers may associated with abnormal BMD in RA. Further long-term follow-up studies should evaluate if high levels of these serum biomarkers could be useful as tests to predict the future development of osteoporotic fractures.

References:

[1] Xu et al. Chin Med J. 2013;126:2480-84.

[2] Xu et al. Rheumatol Int. 2012;32:3397-403.

Acknowledgements: This project was supported by grant from the Fondo de Investigación en Salud del Instituto Mexicano del Seguro Social: FIS/IMSS/PROT/GIS/1455.

Disclosure of Interest: C. Nava-Valdivia: None declared, E. Corona-Sanchez: None declared, J. Ponce-Guarneros: None declared, A. Saldaña-Cruz: None declared, J. Murillo-Vazquez: None declared, E. Perez-Guerrero: None declared, P. Hernandez-Cuervo: None declared, M. Salazar-Paramo: None declared, D. Cardona-Müller: None declared, E. Cardona-Muñoz: None declared, L. Gonzalez-Lopez Grant/research support from: Is a recipient of a Fundación IMSS Scholarship, México., J. Gamez-Nava: None declared DOI: 10.1136/annrheumdis-2017-eular.5652

\section{AB0305 FACTORS CONSIDERED BY RHEUMATOLOGISTS TO DECIDE TREATMENT DURING RHEUMATOID ARTHRITIS PATIENT'S FOLLOW-UP}

C. Sifuentes-Cantu ${ }^{1}$, I. Contreras-Yañez ${ }^{1}$, M. Gutierrez ${ }^{2}$, V. Pascual-Ramos ${ }^{1}$ ${ }^{1}$ Immunology and Rheumatology, Instituto Nacional de Ciencias Médicas y Nutrición Salvador Zubiran: ${ }^{2}$ Division of musculoskeletal and rheumatic diseases, Instituto Nacional de Rehabilitación, México, D.F, Mexico

Background: Disease activity (DA) is the most important factor in the treatment decision during rheumatoid arthritis (RA) patient's follow-up. In routine clinical practice, it is recommended to regularly evaluate DA and musculoskeletal ultrasound has been suggested to add value to establish the level of DA. Nevertheless, the final treatment proposal also requires considering additional factors. In a previous clinical study performed in the setting of an early arthritis clinic, we found that in $20 \%$ of the clinical scenarios evaluated, the German ultrasound score of 7 joints (GUS-7) (1) findings impacted the rheumatologist 's treatment proposal; the impact was more frequent among the trainee in rheumatology (TR) than among the senior rheumatologist (SR).

Objectives: To determine and rate, which among the following factors were determinant to recommend a treatment in the same population of RA patients above described: the clinical assessment, the GUS-7, comorbidities, treatmentrelated adverse events, DMARDs costs/availability, patient's preference and DMARD maximum dose. We also compared if factors differed between both physicians.

Methods: Eighty-seven consecutive and randomly selected RA outpatients were invited to participate; 2 patients denied and 85 patients underwent 170 assessments ( 85 each by the SR and the TR); at first, both physicians (blinded to each other) performed a clinical evaluation that included DAS28 scoring and recommended a RA-treatment. Then, patients underwent GUS-7 by a blinded (to clinical evaluations) rheumatologist. In the final step, the TR and the SR integrated the US findings to their previous evaluation and reviewed their prescription; also, both physicians recorded and rated on a standardized format which of the factors above described were determinant in the final treatment proposal. Patients signed informed consent and were instructed about the process. Only the SR met each patient for the final recommendation. Descriptive statistics was used.

Results: Patients were primarily middle-aged female (91.4\%) and had (mean \pm SD) follow-up of $7.5 \pm 3.9$ years. The majority of them were in DAS28-remission (72\%). Clinical assessment (DAS28) was rated as determinant in the totality of the clinical scenarios (100\%), followed by GUS-7 in $84.7 \%$, DMARD maximum dose in $41.2 \%$, comorbidities in $23.5 \%$, DMARD cost/availability in $21.2 \%$, treatmentrelated adverse events in $20 \%$ and patient's preference in $14.1 \%$ of them. The SR and the TR differed in their selection: GUS-7 and treatment-related adverse events were more frequently considered determinant for the TR $(45.9 \%$ vs. $38.8 \%$, $p=0.01$ and $12.9 \%$ vs. $7.1 \%, p=0.08$ ), meanwhile the opposite figure was true for DMARD cost/availability $(4.7 \%$ vs. $16.5 \%, p \leq 0.001)$ and DMARDs maximum doses $(17.1 \%$ vs. $24.1 \%, p=0.08)$

Conclusions: in a real clinical setting, DA assessed by DAS28 and by musculoskeletal ultrasound were the most important factors to determine the treatment of RA outpatients; additional factors were considered and differently rated by TR and SR.

References:

[1] Backhaus et al. Evaluation of a novel 7-joint ultrasound score in daily rheumatologic practice: a pilot project. Arthritis Rheum. 2009;61: 1194-201.

Disclosure of Interest: None declared

DOI: 10.1136/annrheumdis-2017-eular.1103

\section{AB0306 CAPLAN'S SYNDROME, CADMIUM AND CHINA CLAY: COULD OCCUPATIONAL KAOLIN INHALATION ENHANCE CADMIUM EXPOSURE TO EXPLAIN THE SIXTY YEAR CONUNDRUM OF CAPLAN'S SYNDROME FIRST REPORTED IN COAL MINERS?}

D. Murphy ${ }^{1,2}$, K. Bellis ${ }^{1}$, D. Hutchinson ${ }^{1,2} \cdot{ }^{1}$ Rheumatology, Royal Cornwall Hospital; ${ }^{2}$ University of Exeter Medical School, Cornwall Campus, Truro, United Kingdom

Background: Caplan's syndrome was first described in the coal miners of South Wales (UK). The specific cause for rheumatoid pulmonary nodules associated with coal dust exposure remains unknown. Coal dust exposure alone does not appear to explain Caplan's syndrome as almost all of these men were also smokers. Cigarette smoke is the most important environmental cause of cadmium exposure. We describe Caplan's syndrome in an ex-smoking kaolin worker associated with a significantly raised urinary cadmium level.

Objectives: A 65 year old Cornish kaolin worker and ex-smoker of 20 pack years with life limiting nodular erosive rheumatoid arthritis (RA), developed extra-articular manifestations including pulmonary nodules suggestive of Caplan's syndrome. We review the literature on kaolin, with respect to inhalation and mechanism of action for heavy metal adsorption and nodular disease development.

Methods: Pubmed searches were undertaken using the keywords "kaolinosis", "kaolin", "cadmium" "nodules", "adsorption", "coal". First pass mid-stream urine was analysed for cadmium using inductively coupled plasma-mass spectrometry (ICP-MS). 1

Results: Urinary cadmium was significantly raised at $0.66 \mu \mathrm{mol} / \mathrm{mol}$ creatinine (non-occupationally exposed UK male median $0.17 \mu \mathrm{mol} / \mathrm{mol}$ creatinine ${ }^{1}$ ), 20 years post-industrial exposure and 35 years post-smoking cessation. Contemporary serology levels demonstrated a rheumatoid factor of $481.7 \mathrm{iu} / \mathrm{ml}(0-10$ normal range) and an anti-cyclic citrullinated peptide of $>500 \mathrm{u} / \mathrm{ml}$ (0-17 normal range). CT scan of the chest demonstrated multiple pulmonary nodules consistent with Caplan's syndrome.

The prevalence of Caplan's syndrome in kaolin workers is the highest of any occupation ever reported. ${ }^{2}$ Elevated cadmium levels of $11.2-15.9 \mathrm{mg} / \mathrm{kg}$ have been observed in kaolin, ${ }^{3}$ fifty- fold higher than those reported in coal. ${ }^{4}$ Coal contains variable amounts of clays and minerals such as kaolinite. Cadmium content in coal is strongly associated with levels of kaolinite contamination. ${ }^{4}$ Cadmium 